\title{
Arqueología Protohistórica de la Serena
}

\author{
Sebastián CELESTINO PÉREZ \\ Instituto de Arqueología-Mérida (CSIC) \\ Unidad asociada UAM-IAM
}

\section{Resumen}

Durante la última década, una serie de prospecciones intensivas desarrolladas en la región de La Serena ha permitido comprender mejor la evolución de su paisaje a lo largo de la Historia. Podemos así evaluar mejor el contexto en el que se generaron yacimientos tan significativos como Cancho Roano y otros edificios contemporáneos. Estos estudios han permitido también delimitar las áreas de influencia de centros de control político como Medellín, y la subsiguiente organización del territorio tras la crisis tartésica.

Palabras Clave: Cancho Roano, Tartessos, Prospección intensiva, territorio.

\section{Summary}

Over the last decade a series of intensive archaeological landscape surveys in the 'La Serena' region in the province of Badajoz (Extremadura, Western Spain) has allowed us to better understand the evolution of its lanscape along History. We have also obtained a more balanced view of the context in which archaeological sites as relevant as Cancho Roano and other contemporary buildings were erected and developed. These surveys also allow us to refine our knowledge of the areas of influence of political centres such as Medellin, and the organization of the territory after the crisis of Tartessos.

Keywords: Cancho Roano, Tartessos, intensive survey, territory.

Hace ahora quince años, en julio 1996, el profesor Bendala, acompañado de su mujer, concertó conmigo una visita al santuario de Cancho Roano, donde pasamos una calurosa mañana recorriendo el monumento y discutiendo de los múltiples aspectos del edificio, de las hipótesis que suscitaba su funcionalidad, de las causas de su destruc-

1 Este artículo se enmarca dentro del Proyecto Nacional de I+D HAR2009-10666 "Entre el Atlántico y el Mediterráneo: contraste de dinamicas en la evolucion historica del paisaje ción y, en fin, de cualquier índole de un edificio cuya mejor definición por aquellos años era su enorme complejidad. La discusión se prolongó durante la comida, donde me expuso algunas ideas de interés sobre las posibles influencias que habrían determinado su construcción, así como los acontecimientos que podrían haber influido en

en el occidente peninsular a través de la Arqueologia" cuyo investigador principal es el autor. 
su intencionada destrucción. Al final, sin duda insuflado por los nuevos hallazgos de las construcciones perimetrales, por la incipiente excavación del foso y por el descubrimiento parcial de los dos santuarios más antiguos, se fue con la idea de acometer un artículo sobre los pensamientos que había expuesto, tarea a la que le animé a pesar de la divergencia con algunas de las hipótesis que yo defendía. Sin embargo, nunca llegó a materializar ese trabajo a pesar de la atracción que siempre ha sentido por el yacimiento y su significado. Es verdad que en los últimos cinco años de trabajos en Cancho Roano, de especial intensidad, se habían realizado hallazgos muy significativos y se habían editado cuatro Memorias de excavación y numerosos artículos que obligaron a revisar buena parte de las hipótesis que habían defendido otros investigadores (Celestino 1991,1992, 1997, 2001b; Celestino y Jiménez 1993; Celestino et alii 1996); desde las del propio Maluquer de Motes (1981, 1983; Maluquer et alii, 1987) hasta las que habían expuesto López Pardo (1990) o Almagro-Gorbea $(1989,1990)$. Las excavaciones finalizaron en el año 2001, publicándose en los cinco años siguientes la práctica totalidad de la inmensa documentación generada (Celestino ed. 2003).

A partir de ese momento y a través de diferentes proyectos de los planes nacionales de investigación, comenzó una nueva etapa cuyo principal objetivo estaba destinado a entender el monumento desde el territorio donde se ubicaba y dentro de un paisaje específico que le diera sentido histórico. Ese territorio, en el más amplio sentido del término geográfico, era La Serena, al este de la provincia de Badajoz, una comarca natural donde se plasmaban los hechos arqueológicos más interesantes de la Prehistoria reciente extremeña, desde los tesoros áureos y las estelas del Bronce Final y la I Edad del Hierro (Almagro-Gorbea, 1977; Celestino, 2001b; Rodríguez Díaz y Enríquez, 2001) hasta la romanización a través de los denominados recintos-torre y la cueva del Valle (Rodríguez Díaz y Ortíz, 2003; Mayoral y Celestino ed. 2010; Cazorla y Celestino 2010), pasando por lugares tan señeros de la cultura tartésica como el propio Cancho Roano, Medellín (Almagro-Gorbea, 1977; Almagro-Gorbea (dir.) 2007-2008), La Mata (Rodríguez Díaz (ed.) 2004) u otros poblados y túmulos que caracterizan esta época en la periferia geográfica de Tartessos (Rodríguez Díaz y Ortíz 1998; Jiménez Ávila 1997; Celestino 2005). En los últimos años también se han realizado nuevas excavaciones que han profundizado en el estudio de los asentamientos rurales de la I Edad del Hierro en la Baja Extremadura, donde destaca especialmente el hallazgo, excavación y estudio de Cerro Manzanillo (Rodríguez Díaz, Duque y Pavón (eds.) 2009), un caserío junto a la cuenca del río Ruecas muy cerca de su desembocadura en el Guadiana y dentro de la inmediata área de influencia de Medellín donde se ha podido reconstruir el modelo rural de un asentamiento que funcionó hasta mediados del siglo VI, en pleno periodo Orientalizante.

Todos estos hallazgos se caracterizan tanto por su particularidad arquitectónica como por su riqueza arqueológica debido al alto grado de conservación que presentaban, lo que ha obligado a centrar los trabajos en su completa excavación y en el pormenorizado análisis de su arquitectura y de los numerosos materiales arqueológicos hallados en su interior. Sin embargo, en los últimos años se ha hecho un gran esfuerzo por ampliar los trabajos hacia el territorio que se extiende en torno a los yacimientos protohistóricos más destacados con la intención de entender el significado último de estos destacados yacimientos; así, comenzamos a conocer detalles de gran interés sobre el poblamiento del entorno de Cancho Roano (Walid y Nuño 2005), de La Mata (Rodríguez Díaz et alii 2007), o de Medellín ( Rodríguez Díaz, Pavón y Duque, 2009; Mayoral et alii, e.p.). Esta línea de investigación no ha hecho más que comenzar y, a tenor de los magníficos resultados que se están obteniendo a partir de los diferentes estudios territoriales, creemos que en un futuro próximo estaremos en condiciones de ofrecer un panorama de la zona mucho más acorde con su verdadero significado histórico, donde el interés por la agricultura, por otra parte obvio, y el aumento demográfico detectado a partir del siglo VII, contrasta con un drástico cambio de estrategia política tras la crisis de Tartessos, cuando surge un nuevo sistema de ocupación del territorio y descienden drásticamente los asentamientos rurales que se habían conformado en torno a grandes centros de poder como Medellín. 
En estas páginas expondré los resultados de los últimos trabajos desarrollados desde la Línea de Investigación de Territorio del Instituto de Arqueología del CSIC; con ello, además de aportar un mejor conocimiento del contexto arqueológico en el que se desarrolló Cancho Roano y el territorio donde se ubica, pretendo animar una vez más al profesor Bendala para que con su amplia visión del período histórico en el que se manifestó Cancho Roano contribuya a la discusión, aun abierta, sobre la naturaleza del yacimiento y la evolución del territorio donde se asienta hasta la romanización, donde también se han producido grandes avances en la investigación.

\section{Cancho Roano en SU Contexto terri- TORIAL}

Un año antes de dar por finalizados los trabajos en el yacimiento de Cancho Roano, el entonces Departamento de Prehistoria del Instituto de Historia del CSIC, en colaboración con el Departamento de Geografía y Ordenación del Territorio de la Universidad de Extremadura, puso en marcha un proyecto de investigación centrado en la evolución del paisaje de la comarca de La Serena, donde se ubicaba el yacimiento de Cancho Roano y otros significativos monumentos protohistóricos extremeños. Esta labor, aun hoy en vigor a través de sucesivos proyectos de investigación regionales y nacionales, nació con la intención de conseguir una visión espacial amplia que diera sentido a los procesos económicos y sociales de Cancho Roano, así como a su contexto dentro de un territorio y de una dilatada etapa cronológica. La prospección arqueológica se convirtió así en el método arqueológico más indicado para llevar a cabo ese objetivo, y en concreto la prospección intensiva en el entorno inmediato del santuario; con ello pretendíamos determinar la extensión y la distribución de los materiales, la secuencia en la ocupación de su entorno inmediato, la posibilidad de documentar transiciones en las zonas de actividad agrícola, conseguir información sobre la estructura y función de los asentamientos establecidos en las inmediaciones del santuario y calibrar los factores que podrían haber distorsionado la visión de algunos pequeños sitios arqueológicos en el área estudiada (Mayoral, Cerrillo y Celestino 2009; Mayoral, Cazorla y Celestino, 2010; Celestino y Mayoral 2011).
La primera intervención, entre los años 2000 y 2002, se ciñó a la prospección intensiva del entorno de Cancho Roano, para lo cual se estableció un radio de tres kilómetros a su alrededor para conseguir un registro pormenorizado de los indicios arqueológicos que se organizaban a lo largo del arroyo Cagancha, junto al que se levanta el santuario. La prospección también afectaba a un importante tramo de la vega del Ortigas, río en el que precisamente desemboca el Cagancha a tan sólo $100 \mathrm{~ms}$. al norte de Cancho Roano y de gran importancia estratégica por ser el río que baña buena parte de $\mathrm{La}$ Serena occidental hasta desembocar en el Guadiana, concretamente a la altura de Medellín, uno de los puntos arqueológicos clave para entender la Primera Edad del Hierro de la Baja Extremadura. Aunque los resultados de esta intervención han sido objeto de algunos análisis preliminares (Celestino Pérez y Walid, 2003; Nieto Masot et al. 2003), en la actualidad está en marcha la revisión de la ingente cerámica recuperada en los diferentes sitios localizados en esa prospección, aunque ya estamos en condiciones de avanzar algunos resultados de este estudio. A pesar de que el número de yacimientos localizados en ese radio de tres kilómetros en torno a Cancho Roano es muy elevado, la mayor parte de los mismos corresponden a sitios adscritos a las diferentes etapas de ocupación romana, tanto tardo republicana como Alto y Bajo imperial, así mismo, destacan los sitios pertenecientes a la Edad del Bronce, si bien lo más significativo es la escasa presencia de asentamientos de los últimos momentos del Orientalizante y, sobre todo, la práctica ausencia de sitios prerromanos, algo que ya se ha detectado en otras zonas prospectadas del valle del Guadiana, donde entre los 91 sitios localizados, tan sólo uno puede adscribirse a este período (Rodríguez Díaz, Pavón y Duque, 2009: 187). Entre los hallazgos, pues, destacan algunas villae romanas y restos de caseríos de la misma época asentados en el fértil y bien irrigado triángulo que forman las cuencas del Ortigas y el Cagancha, arroyo éste cuyo cauce de agua no cesa ni tan siquiera en el período estival gracias a los manantiales que lo alimentan, sin duda una de las causas que motivaron la construcción de los sucesivos edificios de Cancho Roano junto a su orilla. 
Tal vez los hallazgos más significativos de estas prospecciones hayan sido algunos de los denominados recintos-torre, pequeñas construcciones fortificadas de época republicana que perviven en época imperial y que tienen una gran presencia en La Serena, aunque no son ajenos a otras áreas del sur peninsular (Mayoral y Celestino (ed.) (2010). Pero también se han hallado pequeños enclaves que coinciden cronológicamente con el desarrollo de Cancho Roano y que demuestran que el santuario no estaba completamente aislado en el paisaje, si bien, y teniendo en cuenta que en los últimos años se ha ampliado sensiblemente el radio de acción de las prospecciones de carácter intensivo, además de haberse realizado varios trabajos de prospección selectiva en buena parte de La Serena, se puede adelantar que no existe ningún poblado de envergadura contemporáneo a Cancho Roano, al menos en este amplio espacio geográfico. No obstante, estamos pendientes de las excavaciones que se están realizando en el castillo de Zalamea de la Serena, donde bajo potentes estructuras romanas de época imperial se han localizado estratos con materiales de importación coetáneos al de Cancho Roano. La ubicación del castillo, dominando buena parte de la vega del Ortigas y del Cagancha, así como fácil comunicación con el yacimiento, situado a menos de tres kilómetros, podría darnos una de las claves para entender la propia dinámica de este territorio en los momentos finales del Periodo Orientalizante.

Para conocer el contexto rural en que se desenvolvió Cancho Roano, hemos recurrido al estudio de algunos de los materiales de los emplazamientos que claramente se podían clasificar dentro de esta época. Se trata de una muestra aún sesgada ya que las piezas escogidas son las que por su morfología, estado de conservación y manufactura nos llevan hacia estos momentos. Especialmente nos hemos centrado en los fragmentos de cerámica gris descubiertos, dado que pueden proporcionarnos una valiosa información en cuanto a la cronología relativa de estos lugares. Se han escogido bordes, bases y asas que por su forma pueden estudiarse dentro de los parámetros de la cerámica gris y más concretamente dentro de los tipos que podemos encontrar en Cancho Roano. Para el resto de fragmentos también nos hemos fijado en los tipos más comunes del yacimiento. De este modo se han seleccionado tres de las áreas catalogadas durante los trabajos de campo: CRA109, CRA144 y CRA169, aunque cabe destacar que no son las únicas que presentan materiales protohistóricos. La prospección se realizó de forma intensiva en un radio de $1 \mathrm{~km}$. del santuario, eligiendo aquellos campos que, al estar recientemente arados, pudiesen tener mayor evidencias de su subsuelo y en donde los restos en superficie estuviesen lo menos dañados posible por efecto de la erosión.

Tras los estudios preliminares, el material recogido en superficie recuerda tipológicamente al común de Cancho Roano: fragmentos de ánforas, abundantes platos grises, vasos de almacén y de mesa, etc. También se localizaron numerosos fragmentos de pizarras como las que adornan los ámbitos más destacados del santuario y que, recordemos, son ajenas a este paisaje. Los tres sitios están ubicados dentro de un radio inferior a un kilómetro del santuario, distancia a la que se encuentra el más alejado de los tres. Todos se hallan junto a orilla del arroyo Cagancha y, precisamente, en el único tramo de este arroyo que, incluso en época estival, mantiene activo el nivel de agua debido a la existencia de un acuífero situado a la altura de uno de los sitios, en concreto el denominado CRA169, precisamente el único que se localizó en la margen izquierda del arroyo. El sitio más alejado de Cancho Roano es CRA109, un pequeño túmulo de no más de $50 \mathrm{~m}$. de diámetro situado en la margen derecha del arroyo Cagancha, a poco menos de $1 \mathrm{~km}$. del santuario; la elevación artificial, de apenas 0,200 ha., está cruzada por una linde donde se ha ido depositando un buen número de piedras de gran tamaño, muchas de ellas trabajadas, a modo de majano o villar. El área CRA144 es una pequeña elevación de apenas 0,15 ha. situada a unos $500 \mathrm{~m}$. al sur de Cancho Roano, donde destaca el abundante y variado material recogido. Por último, el área CRA169 formaba una pequeña elevación a unos 850 ms. también al sur de Cancho Roano, donde se localizó una gran cantidad de cerámica en torno a una pequeña superficie de apenas 0,190 ha.; el lugar, que estaba recién arado en el momento de la prospección, facilitó la recogida de un abundante material cerámico apenas rodado; pero lo más destacado de este sitio es el volumen de material que aportó, así como la calidad de las piezas recuperadas y su variada tipología; destaca, igualmente, la antigüedad aparente del 
yacimiento en sintonía con las fases más antiguas de Cancho Roano.

La profusión y la variedad de la cerámica gris hallada en estos yacimientos han servido para encuadrarlos cronológicamente, aunque también algunos fragmentos de ánforas CR-1 han contribuido al ajuste de la datación (Guerrero 1991). La forma más frecuente que hemos documentado es la perteneciente a los platos de casquete esférico de borde compuesto y labio ligeramente engrosado del subtipo A1C de Lorrio (2008: 679) datados desde la segunda mitad del VII, aunque perduran hasta el primer cuarto del V a.n.e. También destacan por su número los platos de carena media baja con el borde exvasado y el labio redondeado perteneciente al subtipo A3A de Lorrio o al 2D1 de Mancebo (Lorrio 2008: 689; Mancebo 1995: 107); estos platos tienen una mayor recorrido cronológico, pues aparecen hacia los inicios del siglo VII en Medellín pero perduran hasta mediados del siglo $\mathrm{V}$ a.n.e. El resto de las piezas, aunque aun en estudio, se sitúan también sin problemas entre finales del siglo VII y finales del V a.n.e., un dato que confirma la contemporaneidad de los sitios prospectados con las diferentes fases constructivas de Cancho Roano. En este sentido, no debemos olvidar que el edificio más antiguo de Cancho Roano o "CR. C" se data hacia la primera mitad del siglo VI (Celestino 2001b: 36), mientras que la destrucción ritual del último santuario o "CR. A" se llevó a cabo en los últimos años del siglo $\mathrm{V}$ a.n.e.

Como conclusión, podemos decir que el poblamiento rural en torno a Cancho Roano es bastante limitado, ciñéndose a los tres sitios descritos; además, y por los materiales recuperados, parece que estos asentamientos se corresponden más con las fases antiguas del santuario que con el último edificio, fechado en el transcurso del siglo $\mathrm{V}$ a.n.e., lo que de nuevo vuelve a incidir en el aislamiento del santuario en los últimos momentos del Orientalizante, cuando, paradójicamente, muestra su mayor grado de esplendor. Este hecho se antoja de una gran importancia por cuanto responde a unas circunstancias ya detectadas en otros territorios de la denominada periferia tartésica. En efecto, sabemos que Medellín logra su mayor apogeo demográfico entre los siglos VII y VI a.n.e., cuando además ejerce un claro dominio territorial que se plasma en las numerosas granjas localizadas en su inmediato entorno, datadas en consonancia con esos momentos de mayor auge de Medellín (Rodríguez Díaz, Pavón y Duque 2009: 196); por el contrario, a partir de mediados del siglo VI, se detecta en toda La Serena un drástico descenso de este tipo de asentamientos rurales, mientras que surgen los grandes y complejos edificios de evidente significado económico como el de La Mata, que simbolizan un evidente cambio en las relaciones clientelares entre el campo y un centro de poder como fue Medellín. Sin embargo, curiosamente, en las recientes monografías sobre Medellín, los autores proponen el enclave como una ciudad-estado, identificada con Conisturgis (Alarcao, 2001: 337), perteneciente al núcleo tartésico "y de rango estatal" que dominaría un amplio territorio dominado por lo que denominan "palacios-fortines" (AlmagroGorbea y Torres, 2007:53; Almagro-Gorbea et alii, 2008: 1028). Sin entrar a valorar aquí la posible identificación de Medellín con Conisturgis, ni por su puesto la importancia que debió ejercer Medellín como núcleo urbano de referencia sobre un amplio territorio de La Serena en plena época tartésica, sí plantea más problemas considerar este importante enclave junto a uno de los principales vados del Guadiana como el sitio de referencia a partir de mediados del siglo VI a.n.e. Como es sabido, a partir de estas fechas, Tartessos entra en una crisis socio-cultural que repercutirá en su modelo de ocupación del territorio y en sus propias manifestaciones culturales, dando lugar al mundo turdetano en el suroeste.

\section{LA ORGANIZACIÓN DEL TERRITORIO AL final del Periodo Orientalizante}

Como hemos visto, pues, existe en la cuenca media del Guadiana una fase coincidente con el auge de Tartessos en la que podemos hablar de un control de la tierra ejercido desde poblados de grandes dimensiones como Medellín, probablemente organizado y habitado en buena medida por gentes procedentes del núcleo de Tartessos. Alrededor de Medellín se estructuró un extenso territorio agrícola dominado por granjas o caseríos dedicados a la explotación cerealística, sin que hasta el momento se haya detectado en ninguno de ellos pruebas de otros cultivos como la vid o el olivo, ya presentes en el valle del Guadalquivir. Es lógico pensar que la población de estas granjas sea autóctona, por lo que pasaría de una economía 
de subsistencia heredada del Bronce Final a una generadora de excedentes agrícolas. Esta circunstancia permitiría tanto el enriquecimiento del "lugar central" desde donde se ejerce el control de la producción, lo que sin duda transmite la necrópolis de Medellín, así como la estabilidad demográfica de los pequeños centros que lo abastecen. Un sistema, en definitiva, que poco difiere del ensayado en el núcleo tartésico del valle del Guadalquivir, aunque en este caso mucho más desarrollado gracias a la directa intervención de los fenicios.

También la crisis del modelo tartésico afectó a toda su periferia territorial, haciéndose más patente en los núcleos principales, caso de Medellín. Este cambio supuso a la vez la desaparición o abandono de los asentamientos satélites que se habían levantado al amparo y bajo el dominio económico de estos grandes centros; de esta forma, se percibe claramente el desmantelamiento de las granjas que habían surgido en el transcurso del siglo VII y, sobre todo, en la primera mitad del siglo VI a.n.e.; al mismo tiempo, otros asentamientos de mayor calado económico y urbano como El Palomar (Jiménez y Ortega, 2001), también interrumpieron su actividad hacia finales del siglo VI. Medellín no es ajeno a este fenómeno como se advierte en el sensible descenso del número de tumbas que se detecta en su necrópolis a partir de mediados del siglo VI y muy especialmente en el transcurso del V, cuando la presencia de enterramientos es prácticamente residual (Almagro-Gorbea, 2008: 892). Por ello, es difícil entender que sea precisamente en esta última fase del Orientalizante cuando Medellín adquiera la capacidad suficiente para dominar el amplio territorio de La Serena como defienden los autores de su reciente y completo estudio; por el contrario, parece que el modelo que sirvió para que Medellín ejerciera como lugar central del amplio territorio circundante se desmorona hacia finales del siglo VI, a la vez que surge un nuevo fenómeno que se articula también en el paisaje rural de La Serena y que se caracteriza por la construcción de grandes edificios como La Mata de Campanario, cuyo modelo arquitectónico se inspira en Cancho Roano y en otros edificios del área nuclear tartésica.

Estos edificios de piedra y adobe se conservan bajo grandes túmulos que se localizan fácilmente en las inmediaciones del valle del
Guadiana, unas tierras con una innegable vocación agrícola. Lamentablemente, las grandes remociones del terreno como consecuencia del Plan Badajoz de los años 50 del pasado siglo han alterado la distribución de estos túmulos, aunque algunos han sobrevivido a las explanaciones llevadas a cabo para la implantación de arrozales u otras explotaciones agrícolas extensivas; a pesar de ello, los túmulos documentados muestran un nuevo modelo de asentamiento y de control de la tierra hasta esos momentos inéditos, si bien mantienen buena parte de los gustos orientalizantes de la fase anterior. La construcción de estos grandes edificios bajo túmulo se ha interpretado como una "señorialización" del campo (Rodríguez Díaz et alii, 2007: 99) surgida tras la crisis de Tartessos. Lo que parece claro es que el modelo que se desarrolló en el valle del Guadiana en época tartésica, con grandes centros económicos de carácter protourbano donde Medellín representa hasta el momento el mejor, sino el único ejemplo, desaparece de forma abrupta coincidiendo con la crisis tartésica de la segunda mitad del siglo VI a.n.e.; sin embargo, lejos de caer en una profunda crisis como consecuencia del derrumbamiento del sistema que hasta ese momento regía en el área tartésica, la cuenca del Guadiana vivirá a partir de ese momento, finales del VI a.n.e., su época de mayor apogeo económico y cultural, reorganizando las relaciones de poder, la propiedad del campo e introduciendo nuevos cultivos, pero sin abandonar sus estrechos lazos culturales con la fase anterior.

Un caso aparte es, precisamente, Cancho Roano, alejado de las fértiles tierras de la cuenca del Guadiana donde se levantan los grandes edificios bajo túmulo de esta última fase, y construido a tal distancia de Medellín y del resto de túmulos orientalizantes (una media de $45 \mathrm{kms}$ ) que pone incluso en duda su pertenencia al mismo territorio político. Por último, se olvida con frecuencia que el edificio más antiguo de Cancho Roano se construyó hacia finales del siglo VII o inicios del VI a.n.e., en sintonía con la colonización tartésica del interior que algunos defendemos (Almagro-Gorbea 1999: 37; Almagro-Gorbea y otros 2008: 1049; Celestino 2005: 777), lo que significa que su origen está desvinculado de las causas que ocasionaron la nueva ordenación del territorio hacia finales del 
siglo VI, cuando surgen esos edificios de gran complejidad arquitectónica inspirados en el último edificio de Cancho Roano. Por ello, hay que insistir en la funcionalidad estrictamente económica de esos edificios bajo túmulo tipo La Mata, donde sí se localizan pequeñas granjas en su entorno y dibujan un territorio articulado a lo largo de la muy fértil cuenca del Guadiana. Cancho Roano, por el contrario, adolece de un poblamiento estructurado en su entorno y se ubica en un paisaje de bosque que limita su capacidad agrícola; además, como evidencian la distribución de los espacios internos del edificio, así como los tipos y la ingente cantidad de material recuperado, desempeñó una función suprarregional de marcado carácter comercial; mientras que los amplios espacios reservados al culto, los complejos rituales documentados y su propia destrucción no dejan duda sobre su importante función religiosa.

La destrucción de Cancho Roano en los últimos años del siglo $\mathrm{V}$ a.n.e. coincide con la destrucción o incendio de todos los edificios que se conservan bajo túmulo en el valle del Guadiana, lo que se ha venido denominando como la "crisis del cuatrocientos" (Rodríguez Díaz, 1994), un término acertado al menos para esta zona del Guadiana. Pero mientras la mayor parte de estos edificio monumentales fueron abandonados de forma precipitada, en el caso de Cancho Roano se procedió a una destrucción organizada precedida de un complejo ritual (Celestino y Cabrera, 2008) lo que demuestra que las causas de estas destrucciones y del fin del sistema social imperante se debieron a circunstancias externas, tal vez relacionadas con la penetración de gentes desde $\mathrm{La}$ Meseta y su estabilización en el sur de la provincia de Badajoz (Berrocal, 1992). En este sentido hay un dato que se antoja de lo más significativo, pues después de la mencionada destrucción de los últimos edificios orientalizantes a finales del siglo $\mathrm{V}$ o en los primeros años del IV a.n.e., se percibe un abandono del territorio interior de La Serena que aun no ha podido ser bien valorado. En efecto, son bien conocidos los oppida prerromanos diseminados por todo el valle medio del Guadiana, ubicados en cerros junto a la orilla del río y defendidos por murallas (Almagro-Gorbea y Martín (eds.), 1994; Rodríguez Díaz y Enriquez, 2001), sin embargo, el resultado de las prospecciones llevadas a cabo en la comarca de $\mathrm{La}$
Serena, tanto de carácter intensivo como selectivo, han sido incapaces de detectar asentamientos en llano de cierta envergadura, lo que supone una extraña desconexión entre las gentes asentadas junto al Guadiana de las que se desplegaron por el sur de la provincia de Badajoz, donde por el contrario sí se ha detectado un auge poblacional sobresaliente. La causa de este despoblamiento se debió, muy probablemente, al cambio de estrategia económica de la zona, ahora menos preocupada por la agricultura extensiva y más centrada en la explotación minera y el control de las nuevas rutas comerciales. De esta manera, hasta la romanización de la zona, en el siglo II a.n.e., no se vuelve a detectar una intensa ocupación del territorio de La Serena.

La próxima finalización de los diferentes proyectos de investigación que sobre el estudio del territorio de La Serena se están llevando a cabo, nos permitirá afinar mejor sobre todos estos aspectos; en estas páginas sólo he querido hacer una síntesis del estado del conocimiento en el que nos hallamos en estos momentos, avanzando algunos datos y reflexiones para su mejor comprensión.

Madrid, verano de 2011

\section{Bibliografía}

ALARCAO, J. de (2001): "Novas perspectivas sobre os Lusitanos (e outros mundos)" Revista Portuguesa de Arqueologia, 4, 2: 293-349.

ALMAGRO-GORBEA, M. (1977): El Bronce Final y el Período Orientalizante en Extremadura. Biblitheca Praehistorica Hispana, XIV. Madrid.

ALMAGRO-GORBEA, M. (1999): "El territorio de Medellín en época protohistórica" En J.G. Gorges y F.G. Rodríguez (eds.): Économie et territoire en Lusitanie romaine. Collection de la Casa de Velázquez, 65. Madrid: 17-38.

ALMAGRO-GORBEA, M. (2007-2008): La Necrópolis de Medellín Bibliotheca Archaeologica Hispana 26-1,2 y 3. Real Academia de la Historia. Madrid.

ALMAGRO-GORBEA, M. y DOMÍNGUEZ, A. (1989): "El palacio de Cancho Roano y sus paralelos arquitectónicos y funcionales" Zephyrus, XLI-XLII. Salamanaca: 339-382. 
ALMAGRO-GORBEA,M., DOMINGUEZ,A. y LÓPEZ AMBITE, F. (1990): “Cancho Roano. Un palacio orientalizante en la Península Ibérica" Madrider Mitteilungen 31: 251-308.

ALMAGRO-GORBEA,M. y MARTÍN, A,Ma . (eds.) (1994): Castros y oppida de Extremadura. Complutum Extra 4. Madrid.

ALMAGRO-GORBEA,M. y TORRES, M. (2007): "Las fortificaciones tartésicas en el suroeste peninsular" En L. Berrocal y P. Moret (eds.): Paisajes fortificados de la Edad del Hierro. Las murallas protohistóricas de La Meseta y la vertiente atlántica en su contexto europeo. RAHCasa Velazquez. Madrid: 35-55.

BERROCAL, L. (1992): Los pueblos célticos del Suroeste Peninsular. Monografías Extra Complutum, 2, Universidad Complutense, 1992, Madrid.

CAZORLA, R. y CELESTINO, S. (2010): "La cueva del Valle ( Zalamea de la Serena. Badajoz): un santuario rupestre en la comarca de La Serena. SPAL 17: 207-231.

CELESTINO, S. (1991): "Cancho Roano, un complejo urbano orientalizante en Zalamea de la Serena (Badajoz). La presencia del material etrusco en la Península Ibérica. Universidad Barcelona: 439456.

CELESTINO, S. (1992): “Cancho Roano. Un centro comercial de carácter político-religioso e influencia oriental". Rivista di Studi Fenici XX-1. Roma: 19-46.

CELESTINO, S. (1994): "Los altares en forma de lingote chipriota de los santuarios de Cancho Roano". Revista de Estudios Ibéricos, 1. Universidad Autónoma de Madrid: 291-309.

.CELESTINO, S. (ed.) (1996): El Palacio-Santuario de Cancho Roano V-VI-VII. Los sectores oeste, sur y este., Junta de Extremadura., Publicaciones del Museo Arqueológico Provincial de Badajoz, 3. Badajoz.

CELESTINO, S. (1997): "Santuarios, centros comerciales y lugares sacros" Espacios y lugares cultuales en el mundo ibérica. Quaderns de Prehistòria i Arqueologia de Castelló, 18; 359390.

CELESTINO, S. (2001): Estelas de guerrero y estelas diademadas. La precolonización y formación del mundo tartésico. Bellaterra. Barcelona.

CELESTINO, S. (2001b): "Los santuarios de Cancho Roano: del indigenismo al orientalismo arquitectónico" en Ruiz Mata, D. y Celestino Pérez, S. (coords.): Arquitectura oriental y orientalizante en la península ibérica. CSIC-Centro de Estudios del Próximo Oriente-Instituto de Historia. Madrid; 17-56.

CELESTINO, S. (ed.) (2003): Cancho Roano VIII-IX. Los materiales arqueológicos, Instituto de Arqueología del CSIC. Mérida.

CELESTINO, S. (2005): "El Periodo Orientalizante en Extremadura y la colonización tartésica del interior" En S. Celestino y J. Jimenez (eds.). El Periodo Orientalizante. Actas del III Simposio Internacional de Arqueología de Mérida: Protohistoria del Mediterráneo Occidental. Anejos de Archivo Español de Arqueología XXXV. Madrid: 767-786.

CELESTINO, S.; CABRERA, A. (2008): "El banquete privado y el banquete comunal en el santuario de Cancho Roano" Cuadernos de Prehistoria y Arqueología, 18: 189-215

CELESTINO, S. y JIMÉNEZ J. (1993) El PalacioSantuario de Cancho Roano IV. El sector norte, B. Gil Santacruz. Badajoz.

CELESTINO, S. y MAYORAL, V. (2011): "Intensive survey and protohistoric settlements in the middle Guadiana basin (Badajoz, Spain)" Hidden Landscapes of Mediterranean Europe. Cultural and methodological biases in pre- and protohistoric landscape studies. Universidad de Siena. Siena.

CELESTINO, S., WALID, S. (2003): "Proyecto arqueológico "La Serena". En S. Torallas ed. Memoria. Seminario de Filología e Historia. CSIC. Madrid: 47-53.

GUERRERO, V. (1991): “El palacio-santuario de Cancho Roano (Badajoz) y la comercialización de ánforas fenicias indígenas" Rivista di Studi Fenici XIX-1: 49-82

JIMÉNEZ AVILA, J.(1997): “ Cancho Roano y los complejos monumentales post-orientalizantes del Guadiana" Complutum, 8, p. 141-160.

JIMÉNEZ AVILA, J. y ORTEGA, J. (2001): "El poblado orientalizante de El Palomar (Oliva de Mérida. Badajoz). Noticia preliminar" En D. Ruiz Mata y S. Celestino (eds.) Arquitectura oriental y orientalizante en la Península Ibérica. CEPOCSIC. Madrid: 227-248.

LÓPEZ PARDO, F. (1990): "Sobre la función del edificio singular de Cancho Roano (Zalamea de la Serena, Badajoz)" en Gerión, 8: 141-162.

LORRIO, A. J. (1989): “Cerámica gris orientalizante de la necrópolis de Medellín (Badajoz)", Zephyrus, XLI-XLII, Salamanca, PP. 283-314. 
LORRIO, A. J. (2008): "La cerámica gris" en Almagro-Gorbea (dir.) La Necrópolis de Medellín. II. Estudio de los hallazgos. Bibliotheca Archaeologica Hispana 26-2 R.A.H. Madrid: 673-723

MANCEBO, J. (1995): "La cerámica gris a torno de la campiña sevillana”, XXII Congreso Nacional de Arqueología, vol. 1, (Vigo 1993), Vigo, pp. 177-181.

MALUQUER DE MOTES, J. (1981): El santuario protohistórico de Zalamea de La Serena, Badajoz. Vol. I, 1978-1981. Programa de Investigaciones Protohistóricas IV. Institución Milá y Fontanals, Universidad de Barcelona. Barcelona.

MALUQUER DE MOTES, J. (1983): El santuario protohistórico de Zalamea de la Serena (Badajoz). 1981-1983. Programa de Investigaciones Protohistóricas, V, CSIC, Barcelona.

MALUQUER DE MOTES, J., CELESTINO, S., GRACIA, F. y MUNILLA, G. (1986): El Santuario protohistórico de Zalamea de la Serena (Badajoz). 1983-1986. Programa de Investigaciones Protohistóricas, XVII, CSIC, Barcelona

MAYORAL, V., BORJA, F., BORJA, C., , MARTÍNEZ, J.A., y DE TENA, M.T. (e.p.): "The evolution of an agrarian landscape. Methodological proposals for the archaeological study of the alluvial plain of Medellin (Guadiana basin, Spain)" Proceedings of the first international Landscape Archaeology Conference, 26$28^{\wedge}$ th January 2010. Amsterdam.

MAYORAL, V., CAZORLA, R. y CELESTINO, S. (2010): "The Romanization process of an agrarian landscape: La Serena región". En C. Corsi y F. Vermeulen eds. Changing Landscapes. The impac of Roman towns in the Western Mediterranean. Ed. Ante Quem. Bolonia: 263279.

MAYORAL, V. y CELESTINO, S. (Coords.) (2010): Los paisajes rurales de la romanización. Arquitectura y explotación del territorio. Ed. La Ergastula. Madrid.

MAYORAL, V., CERRILLO, E. y CELESTINO, S. (2009): "Métodos de prospección arqueológica intensiva en el marco de un proyecto regional: el caso de la comarca de La Serena (Badajoz)". TP, $66, \mathrm{n}^{\circ} 1 ; 7-26$.
NIETO, A., HERNÁNDEZ CARRETERO, A. y LECO, F. ( 2003): Metodología de prospección arqueológica mediante imágenes Landsat TM y Spot PAN en Zalamea de la Serena (Badajoz). IX Conferencia Iberoamericana de Sistemas de Información Geográfica. Cáceres.

ORTIZ, P. y RODRÍGUEZ DÍAZ, A. (1998): "Culturas indígenas y romanización en Extremadura: castros, oppida y recintos ciclópeos" en Rodríguez Díaz, A. (coord.): Extremadura protohistórica: paleoambiente, economía y poblamiento. Universidad de Extremadura. Cáceres; 247-278.

RODRÍGUEZ DÍAZ, A. (Ed.) (2004): El edificio protohistórico de "La Mata" (Campanario, Badajoz) y su estudio territorial. Universidad de Extremadura. Cáceres.

RODRÍGUEZ DÍAZ, A, DUQUE, D.M. y PAVÓN (eds) (2009): El caserío de Cerro Manzanillo (Villar de Rena, Badajoz) y la colonización agraria orientalizante en el Guadiana Medio. Memorias de Arqueología Extremeña, 12. Mérida.

RODRÍGUEZ DÍAZ, A. y ENRÍQUEZ, J.J. (2001): Extremadura tartésica. Arqueología de un proceso periférico. Ed. Bellaterra. Barcelona.

RODRÍGUEZ DÍAZ, A. y ORTIZ, P. (2003): “Defensa y territorio en la Beturia: castros, oppida y recintos ciclópeos" en Morillo, A.; Cadiou, F.; Hourcade, D. (coords.): Defensa y territorio en Hispania de los Escipiones a Augusto: espacios urbanos y rurales, municipales y provinciales. Universidad de León-Casa de Velázquez. León; 219-252.

RODRÍGUEZ DÍAZ, A., PAVÓN, I., DUQUE, D.M. y ORTIZ, P. (2007): "La señorialización del campo postartésica en el Guadiana Medio: el edificio protohistórico de La Mata (Campanario. Badajoz) y su territorio" En A. Rodríguez e I. Pavón (eds.): Arqueología de la tierra. Paisajes rurales de la protohistoria peninsular. Universidad de Extremadura. Cáceres: 71-101.

WALID, S. y NUÑO, R. (2005): “Aplicaciones arqueogeográficas al estudio de las sociedades del período orientalizante. ¿Quién construyó Cancho Roano?" en Celestino Pérez, S. y Jiménez Ávila, J. (eds.): El Período Orientalizante. Actas del III Simposio Internacional de Arqueología de Mérida: Protohistoria del Mediterráneo Occidental. CSIC; Mérida; 977-984. 
